\section{Indecisive interleukin-4?}

A study on page 1899 may explain the seemingly fickle ways of the cytokine interleukin (IL)-4. Yao and colleagues show how this quintessential T helper (Th) 2 cytokine can sometimes promote the opposite Th1 response.

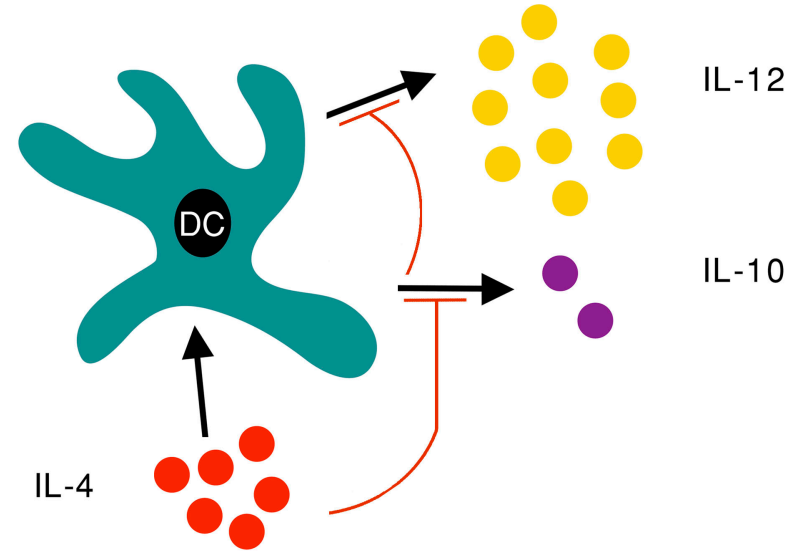

The prototypic Th2 cytokine IL-4 inhibits IL-10 production by DCs, thereby increasing the production of the Th1-promoting cytokine IL-12.
$\mathrm{IL}-4$ is known as the key cytokine for polarizing naive T cells toward a Th2 phenotype, which is important for antibody production and protection against parasitic infections. Under some conditions, however, IL-4 has been shown to instead induce a Th1 response. Indeed, a recent study showed that mice treated with IL-4 during initial infection with Leishmania major had increased Th1 responses and were protected. If given later, IL-4 increased Th2 responses and exacerbated disease.

Yao et al. now suggest that the regulation of another cytokine-IL-10-may explain these perplexing observations. They show that dendritic cells (DCs) stimulated in the presence of IL-4 made less IL-10 than those stimulated without IL-4. As a result, the IL-4-treated DCs produced more of the Th1-polarizing cytokine IL-12-known to be inhibited by IL-10-and polarized naive T cells toward a Th1 phenotype more effectively. IL-10 was critical for the increased Th1 response, as IL-4 did not increase IL-12 production or T cell polarization by IL-10-deficient DCs.

IL-4 had the opposite effect on B cells, provoking increased IL-10 production. The authors suggest that the differential effect of IL-4 at different times during infection may reflect a switch from DCs to B cells as the predominant cell type that is presenting antigen. JEM

\section{Lymphocytes that can't let go}

On page 1987, Semmrich and colleagues show that immune cells expressing a perpetually activated form of the integrin LFA-1 get traction at the front of the cell, but get stuck from behind. Their lagging ends prevent them from crawling through the endothelium and initiating a normal immune response.

Integrins, such as LFA-1, are adhesive molecules that switch between active and inactive conformations and control migration of circulating immune cells to sites of infection and inflammation. LFA-1 is also required for the formation of stable interactions between $\mathrm{T}$ cells and antigen presenting cells (APCs). Previous studies had shown that both tumor-specific $\mathrm{T}$ cell responses and neutrophil migration are compromised in the absence of this integrin.

The importance of LFA-1 deactivation, however, has been less clear. In vitro studies have shown that locking LFA-1 in its activated conformation impairs both neutrophil chemotaxis and $\mathrm{T}$ cell activation. Semmrich et al. now confirm these findings in vivo, and show that lymphocytes from mice expressing a constitutively active LFA-1 moved more slowly than wild-type cells and failed to migrate across endothelial cell monolayers. Video microscopy revealed that these defective movements were due to an inability of the cells to release their trailing edges.

$\mathrm{CD}^{+}{ }^{+} \mathrm{T}$ cell proliferation and antibody production were also impaired in these mice. The development of antigenspecific $\mathrm{CD}^{+}$cytotoxic $\mathrm{T}$ cells, by contrast, was not altered by the mutated LFA-1, but the ability of these cells to lyse target cells was decreased. The authors suggest that defects in $\mathrm{T}$ cell activation and function may reflect the need for serial engagement of $\mathrm{T}$ cell receptors with peptide-MHC complexes on APCs, a process that may be compromised by the inability to inactivate LFA-1 and thus to terminate cell-cell contacts.

Mice expressing the mutant LFA-1 closely resembled mice lacking the protein completely, suggesting that, for immune cells, letting go is just as important as grabbing on. JEM
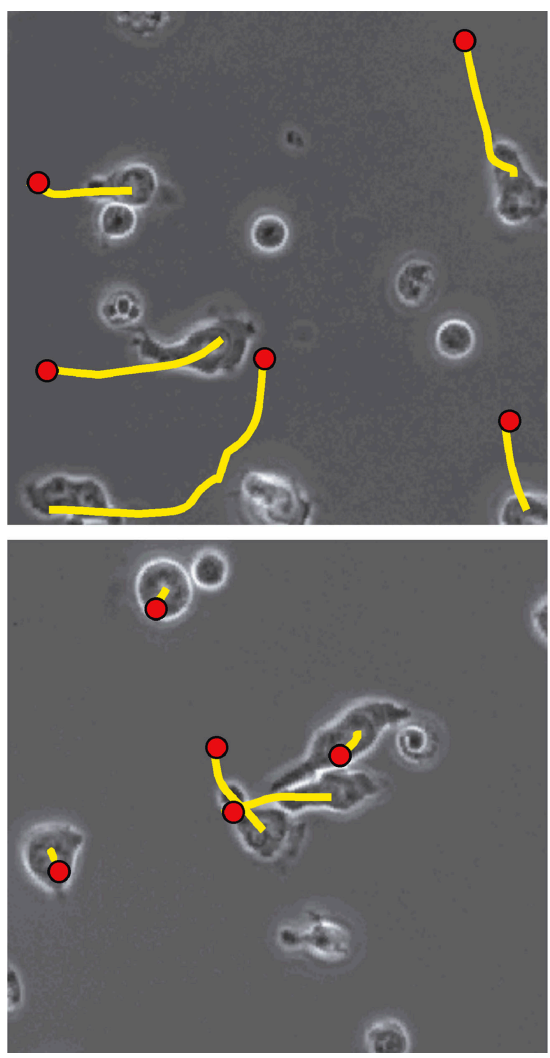

T cells that express a constitutively activated form of the integrin LFA-1 (bottom) have restricted mobility compared to those expressing wild-type LFA-1 (top). 